\title{
sciendo
}

Transport and Telecommunication, 2022, volume 23, no. 1, 33-47

Transport and Telecommunication Institute, Lomonosova 1, Riga, LV-1019, Latvia

DOI 10.2478/ttj-2022-0004

\section{RELIABLE AND SEAMLESS COMMUNICATIONS OF NETWORKED IAV IN CONTAINER TERMINAL USING UAV TECHNOLOGY}

\author{
Nacera Bahnes ${ }^{1,2}$, Bouabdellah Kechar ${ }^{1}$, Hafid Haffaf ${ }^{1}$ \\ ${ }^{1}$ Industrial Computing and Networks Laboratory (RIIR) \\ Computer science Department, University of Oran 1 Ahmed Ben Bella \\ PO Box 1524 El M'naouar, Oran - Algeria \\ ${ }^{2}$ Computer sciences Department, University of Abdelhamid Ibn Badis - Mostaganem \\ 27000, Mostaganem - Algeria \\ bahnes_nacera@yahoo.fr
}

\begin{abstract}
Managing container loading and unloading operations at a container terminal using Intelligent Autonomous Vehicle (IAV) is challenging, especially at intersections in the yard, which are often inevitable. For ensuring efficient and accident-free management of these intersections, the IAV must cooperate by exchanging messages. Due to signal obstruction at these intersections, indirect communication is established through an additional relay node to ensure reliable communication. This paper proposes distributed approach using Unmanned Aerial Vehicle (UAV) connectivity to avoid collision and deadlock between IAV at intersections. Due to the obstacles formed by stacked containers blocking the radio transmissions, the proposed algorithm automatically switches the ongoing communication through the UAV to ensure successful communication at intersections. Thus, the idea of introducing UAV as communication relay in a container terminal is an interesting solution that we have adopted in this work. Simulation techniques are used to evaluate our proposal. The obtained results confirm that our UAV-based approach ensures reliable communication and automated intersections management in the yard while further ensuring the safety of IAV traffic.
\end{abstract}

Keywords: UAV, wireless communication, IAV, intersection, ACT, distributed algorithm

\section{Introduction}

Container terminal (CT) is a complex transportation system (Guenther et al., 2006). Its primary function is container handling, which includes loading, unloading, horizontal movement, sorting, and storage of containers in the yard. Efficient management of terminal operations and equipment requires advanced planning and control system. Several cranes and vehicles equip the CT for vertical and horizontal transportation. Automation of container terminals has recently become a strategic trend where unmanned equipment and advanced technologies are presently being used in the container terminal (URL1, 2021). Scheduling and controlling these kinds of unmanned equipment is an extremely important and difficult task. Advanced technologies such as sensor networks, the Internet of Things (IoT), and wireless communication are used to solve automated handling problems, increase terminal efficiency, and reduce vessel unloading/loading time. Wireless communication is gradually becoming more used in several areas with connected nodes. Each connected node (static or mobile) transmits data to other nodes through radio waves. In Vehicular Ad-hoc NETwork (VANET), Vehicle-to-Vehicle (V2V) communication improves road traffic safety and assists the automated movement of vehicles. Indeed, each vehicle determines locally neighbor vehicles through the transmission and reception of periodic messages (beacon messages). These exchanges could be shown to be effective, and accordingly, it is a key factor for the success of cooperation tasks among all connected nodes. There are several reasons why the transmission of safety messages to neighboring vehicles might fail, such as fading, shadowing, congestion, interference...etc. The direct communication link between connected vehicles is often blocked by obstacles such as buildings, vegetation, and parked or moving vehicles with the signal obstruction. This does not necessarily result in packets loss but still leads to considerable signal attenuation. Radio waves attenuation negatively affects the quality of communication links. Belmekki et al. (2019) studied communication between vehicles at intersections by considering the Nakagami-m fading channel, and they proved that the relay transmission was completing the direct transmission. In this situation, indirect communication is useful. Especially in the CT, metal objects, such as piles of stacked containers and equipment, significantly impact wireless communication between moving vehicles in the yard. With the signal obstruction, the radio transmission may be totally or partially blocked (see for instance Ambroziak and Katulski, 2014). So, the connected vehicles cannot detect all their 
neighbors most of the time. Bahnes et al. (2016) have proposed a cooperation approach based on wireless communication between IAV to manage the intersection in an automated container terminal (ACT). In this previous work, they have not considered the signal obstruction caused by steel objects. They suppose that direct communication links are almost available within the IAV communication range. In this study, we propose an extension of the cooperation scheme proposed in (Bahnes et al., 2016) by integrating into the communication architecture an unmanned and aerial vehicle (UAV) as dynamic relay to ensure reliable communication between IAV, especially upon approaching an intersection. The main objective of this study is to improve the performance of the IAV cooperation under difficult communication conditions based on wireless communication using the flying node (UAV) as dynamic relay at intersection. The effectiveness and necessity of this approach are studied using extensive simulations. The contributions of this paper are summarized as follows: 1) Contrary to the previous work, we have taken into consideration the signal obstruction caused by the presence of steel objects in the yard. 2) UAV are used in port environments to relay control messages with better transmission conditions and improve the connectivity and efficiency of the IAV network in the ACT. 3) Inter-IAV communication is established in the direct link (1-hop) and/or indirect link (2-hops). 4) Local intersection management is achieved by the proposed cooperation mechanism through wireless communication with immediate neighbors and hidden neighbors in case of necessity. Cooperation is based on network connectivity and continuous communication between IAV. There is a strong need for local control and management of autonomous vehicles in the port by considering signal propagation conditions.

The remainder of this paper is organized as follows. We present the background and related work in Sections 2 and 3, respectively. Section 4 describes the model of an ACT. Our proposal is detailed in Section 5. In Section 6, we analyze the simulation results. Finally, Section 7 concludes this paper.

\section{Background}

This section describes the concepts of IAV, ACT, and UAV, which are the key elements of the proposed solution in this paper. ACT adopts unmanned equipment such as IAV to enhance seaport management. Wireless communication between IAV and UAV form an ad-hoc network. An IAV is an automated vehicle used for the horizontal handling of containers in the future ACT.

\subsection{Automated Container Terminal (ACT)}

The concept of ACT is introduced by the launch of the ECT (Europe Container Terminal) Delta Terminal in the port of Rotterdam in the Netherlands in 1993 (Martin et al., 2014). An automated seaport is safer than the conventional one (e. g URL2 (2020); Chu et al., 2018). ACT equipment is divided into three main categories: i) Quay Cranes (QCs) which are responsible for loading and discharging containers from and to vessels on the quayside; ii) Unmanned vehicles for horizontal transportation such as the Automated Guided Vehicle (AGV) and IAV which are responsible for taking containers from the quayside and transporting them to the storage yard during the discharging operation, and from the storage, yard to quayside during the loading operation; iii) The Automated Stacking Cranes (ASC) which are responsible to handling containers received from the AGV when they arrive at storage yard or to taking containers from container stacks to AGVs during the charging operation. The presence of containers and equipment made of steel causes a very multipath effect. The container terminal should be treated as a different RF propagation environment (Yu et al., 2019).

\subsection{IAV overview}

Intelligent Autonomous Vehicle (IAV) is a new unmanned and electric vehicle developed for horizontal transport in an ACT (Gelareh et al., 2013). IAV plays an important role in the small-scale container terminal and on a large scale for container handling. Contrary to AGV, IAV can move freely in the layout. It is responsible for taking containers from the quayside and transporting them to the storage yard during the discharging operation and from the storage yard to the quayside during the loading operation (Kechar and Haffaf, 2012). On the other hand, the free movement of IAV enhances transportation efficiency, but it could increase traffic complexity which needs more control.

\subsection{UAV for wireless communication}

Unmanned Aerial Vehicle (UAV), mainly named Drone, comes in various sizes and types, with the largest use for military purposes (Hassanaliam and Abdelkafi, 2017). A quadrotor is an example of a UAV with four rotors for its lift and its hover (URL3, 2020). UAV have a wide range of applications and models. UAV are becoming an important part of the transportation system, and they have a major impact 
on public safety. UAV-assisted applications are proposed in many studies (e.g. Dong et al., 2014; Shi et al., 2018). Erdelj et al. (2017) present a survey of UAV-assisted network which describes meteorological disasters management based on the interaction between the UAV and wireless sensor network. UAV aided localization algorithm for the train-mounted mobile terminal is studied in ( $\mathrm{Yu}$ et al., 2019). Cooperation among UAV and unmanned ground vehicles is described in (Melin et al., 2015) to improve the path planning task. Ranjan et al. (2018) propose UAV based multi-hop emergency communication system in deep open-pit mines. In addition, the use of UAV ensures a reliable path selection to increase the efficiency of packet delivery, decrease the end-to-end delay and packets loss ratio (e. g. Oubbati et al., 2015; Oubbati et al., 2016). With the recent advanced technologies of control and wireless communication, UAV is also used in a confined space (Vasarhelyi et al., 2018; Floreano et al., 2015; Noor et al., 2018). UAV is used in CT for inspection, mapping and surveying, monitoring, emergency response, security missions, damages prevention, coverage network, and connectivity. A major advantage of using an UAV as a relay is that it is quickly deployed into the battlefield or in various communication environments and thus improves the performance of the wireless communication system in signal obstruction. Furthermore, the container terminal is a dynamic and distributed platform that requires the use of a relay flying node to enhance the reliability of wireless communication.

\section{Related work}

An intelligent transportation system (ITS) is an application set of sensing, analysis, control, and communication technologies to improve safety, mobility, and efficiency (Lim et al., 2017). ITS will become an umbrella that covers a wide range of transportation systems. Cooperation between connected vehicles is needed for the proper performance of safety and traffic efficiency applications. Automated Intersection Management (AIM) could be one of the best options for controlling access to the conflict zone in real time, avoiding collision, improving traffic efficiency, and minimizing the wait time of all present vehicles at intersections. In conventional traffic, drivers move on the roads and intersections by applying the instructions indicated on the roadside signs. Consequently, an automated vehicle requires similar data to navigate without accidents. With the absence of road signs, the safety traffic requires that vehicles be aware of the presence of nearby vehicles. For the AIM, several approaches and solutions are proposed for both signalized and non-signalized intersections. In (Dresner and Stone, 2004), the authors proposed a global server that controls and organizes the passage of vehicles at an intersection. Other studies use a fixed node as a manager, determining the access sequence of vehicles (see for instance Lombard et al., 2016). The goal is to find the optimized sequential traffic of all AGVs at the intersection in an industrial environment. Moreover, a game theory-based algorithm was given by Elhenany et al. (2015) to control the movement of autonomous vehicles equipped with Cooperative Adaptive Cruise Control (CACC) systems at uncontrolled intersections. Other studies proposed biologically-inspired approaches to self-organize traffic control schemes at the intersections, such as in (Tonguz et al., 2016), where distributed management method is proposed to resolve the potential conflicts at intersection and determine the priority scheme that assigns the priority to specific roads. Ahmane et al. (2013) proposed a traffic control at intersections where the vehicles negotiate wirelessly the "right way" according to the measurements done by the positioning system during their travel. The local management of intersections can be resolved by implementing cooperation or coordination between automated vehicles (e.g. Bahnes et al., 2016; Azimi et al., 2012; Hafer et al., 2013; Campos et al., 2017). All these researches investigated solutions for the automation of intersection management in a centralized or distributed manner without considering the communication problems due to the signal obstruction. Other research works are published to resolve the obstructed direct link between vehicles at intersections, such as relay node based on fixed relay (Yang et al., 2004), barking vehicle, or moving vehicle (e. g. Sommer et al., 2014 ; Echhoff et al., 2011). Multi-hop relaying by neighbor vehicles has been demonstrated to perform well dissemination messages with the presence of obstacles. The multi-hop data dissemination is one of the VANET advantages for extending the transmission of safety and emergency warning messages, exchanging neighborhood information queries, or relaying data to connected nodes in the network (Chen et al., 2011). However, there are likely a few vehicles available as relays on the roads in the low-density traffic zone or at night. In recent scientific research, the possibility of using a UAV as a relay node has begun to appear (e.g. Ruban et al., 2007; Oubbati et al., 2015; Oubbati et al., 2016). The communication network based on UAV is foreseen in several fields (Sahingoz et al., 2013; Yu et al., 2019). In Yang (2016), the authors proposed a new approach based on UAV during its autonomous fly to solve this kind of problem by considering the location information through GPS. Extend the usage of UAV as additional node in the network ensures the safety of automated vehicles, especially at the intersections where direct communication is impossible in the case of communication obstacles that disrupt the signal propagation. 
The deployment of UAV is really easy and facts with low cost. Compared with conventional static relaying, flying nodes offer a new degree of freedom for performance enhancement. On the other hand, installing an additional node is not almost possible in several untypical environments such as container terminals. The latter are considered as very difficult radio waves propagation environments due to the presence of metallic objects.

\section{System model and problematic}

An Automated Container Terminal (ACT) mainly operates with three handling processes: containership operations, container handling, storage operations in the yard, and receiving/delivery operations for road trucks. Containership operations include dispatching and loading/unloading operations. As depicted in Figure 1, three handling equipment types are presented in an ACT: Automated Stacking Crane (ASC), Intelligent Autonomous Vehicle (IAV), and Quay Crane (QC). ASC is used for vertical handling in the storage yard. IAV is then autonomous vehicle used for horizontal transportation. In our model, an intersection is the crossing of two roads. Furthermore, it requires control access if the crossroads have a direction to this intersection. The problem of detection and avoidance collision at intersections is one of the first problems that should be addressed in the free movement of autonomous vehicles in an ACT. As presented in Figure 1, IAV $\mathrm{IA}_{\mathrm{A}}$ and $\mathrm{IAV}_{\mathrm{B}}$ move on different roads and reach the same intersection. So, both IAV are approaching the same intersection. In this case, we say that two IAV could be in a deadlock or collision state.

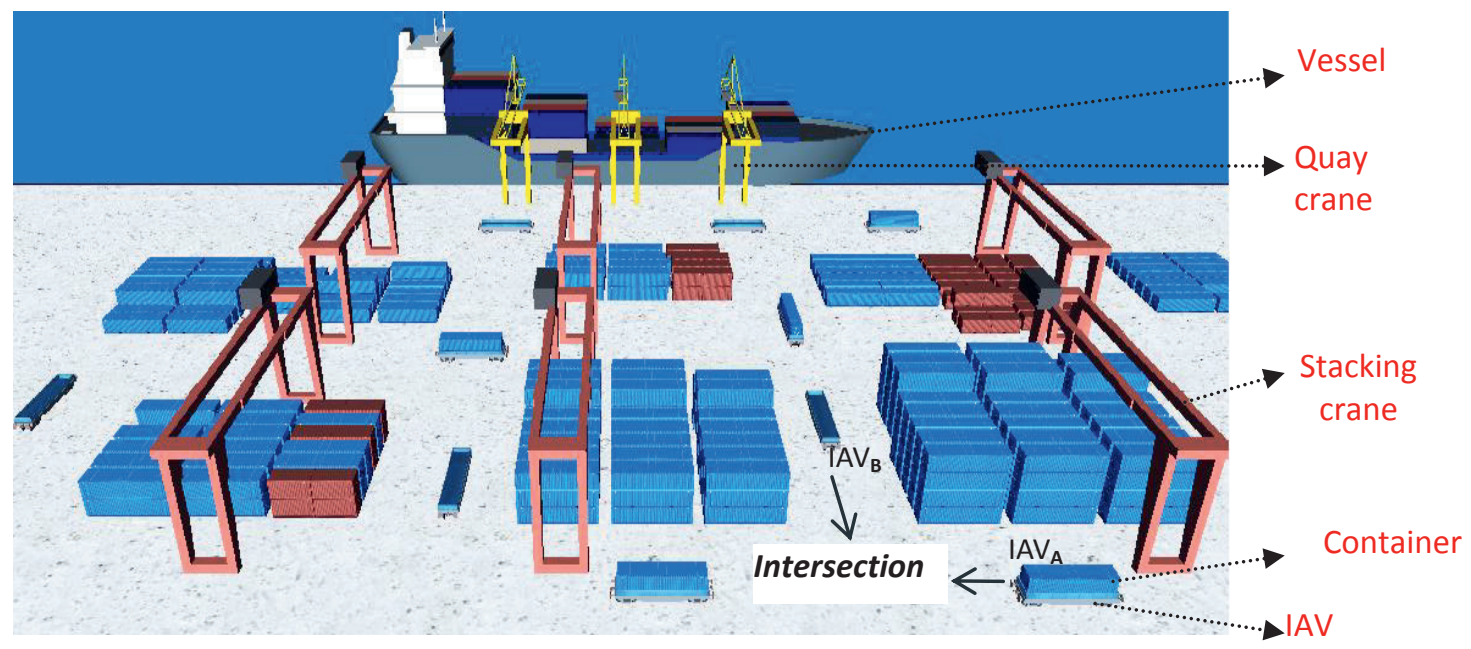

Figure 1. Illustration of a typical ACT with intersections

Bahnes et al. (2016) proposed a new cooperative approach based on wireless communication to manage the intersection access locally without considering the obstruction of the transmitted signal. In this previous study, we assumed that signal propagation in the yard is perfect, which is not always true in reality. The blocks of stacked containers in the storage space are considered as metallic obstacles for wireless communication. In this situation, the control of IAV navigation is required along the roads, especially at the intersections where $\mathrm{V} 2 \mathrm{~V}$ communication is not guaranteed due to signal obstruction. This fundamental problem is our main objective in this work. Indeed, in the present paper, we extend our previous work on intersection management and check the influence of the flying nodes in the sky, which are already placed in advantageous positions.

\section{Design of the proposed solution}

As mentioned above, a cooperation approach based on Inter-IAV communication was first proposed in (Bahnes et al., 2016) to automate the decentralized management of intersections. In the case of signal obstruction, the problem of collision between IAV at intersection needs to be taken seriously. To overcome this issue, an additional node should be used to relay disconnected IAV before reaching intersections. The relay node can be located at any free space in the geographical area around the IAV. For security reasons, it is impossible to install a relay node in the yard area. Precisely for this reason, 
UAV aided cooperation scheme aims at providing collision prevention between the IAV. So, we consider a relay strategy based on UAV as a dynamic relay to assure reliable communication in the ACT. This research aims to take advantage of the UAV to re-establish wireless communication links in case of disconnection due to the obstacles (stacked containers), as depicted in Figure 2. In addition to direct communication between the IAV, indirect communication is supported by UAV deployed on the intersection. The relay transmission is established only when the direct transmission is not possible. The proposed solution is more detailed in the following sub-sections.

\subsection{The use of UAV in our scenario}

Wireless communication based on UAV is the proposed solution where the UAV hovers over the intersection in the storage yard. As a dynamic relay, UAV can assist IAV to solve the problem of disconnection, which frequently occurs in the ACT. As shown in Figure 2, the stacks of containers block direct transmission between IAV. We denote $\boldsymbol{d}$ as the distance between IAV and the intersection (see Figure 2). For example, the direct communication link between "IAV " and "IAV ${ }_{2}$ " is affected by the signal obstruction due to containers' presence between them. IAV collision and deadlock should be prevented to avoid substantial production interruptions. So, when the distance ( $\mathrm{IAV}_{2}$, intersection) is less than $\mathbf{d}, \mathrm{IAV}_{2}$ starts the control phase is from $\boldsymbol{d}$ distance. The control and cooperative phase is based on network connectivity and continuous communication between IAV. In our case, reliable communication is necessary to ensure cooperation between IAV to avoid the blockage at the intersection level. Prevent and avoid collision contribute to the continuance of service in ACT. With the signal obstruction, UAV assists IAV to stay connected with their hidden neighbors and enhances connectivity at intersections.

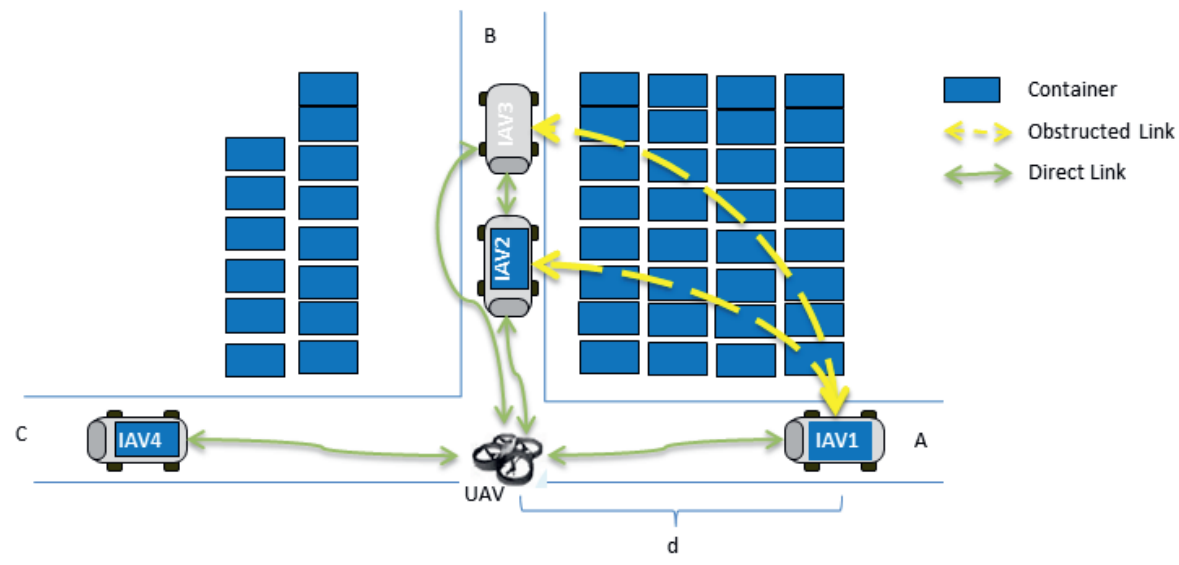

Figure 2. Solution design

\subsection{Intersection scenario}

For this study, two layouts are defined. The first layout is formed as a single loop of a unidirectional route without intersection. In this layout, collision and deadlock at the intersection could not occur. The second layout is formed by a set of unidirectional paths (two horizontal lanes and six vertical lanes), as shown below in Figure 9. The intersection box is the crossing area of a vertical road with a segment of horizontal road. According to our proposition, the intersections are classified into two classes (class A: intersections with the possibility of IAV crossing and class B: other intersections). At the base of roads' direction presented in Figure 9 , only the intersections $\mathrm{P}_{1}, \mathrm{P}_{3}, \mathrm{P}_{7}$, and $\mathrm{P}_{9}$ are in class $\mathrm{A}$ which require control access.

\subsection{IAV and UAV behavior}

Besides handling containers, the IAV communicates with neighbor nodes (IAV and UAV) in the yard by sending and reception of beacon messages, control messages, and replies of control messages. Transmission of these messages serves to update neighbors' list and guarantees safety access at the intersections. Figure 3 shows different IAV states in communication task according to the sequence of transmitted messages, which is depicted in Figure 5. Each UAV updates its neighbor's list using the received data and periodically sends it to the IAV in its range communication. The UAV plays the role of an intermediate node between the IAV to accomplish the control operation. The UAV states in its communication model are shown in Figure 4. 


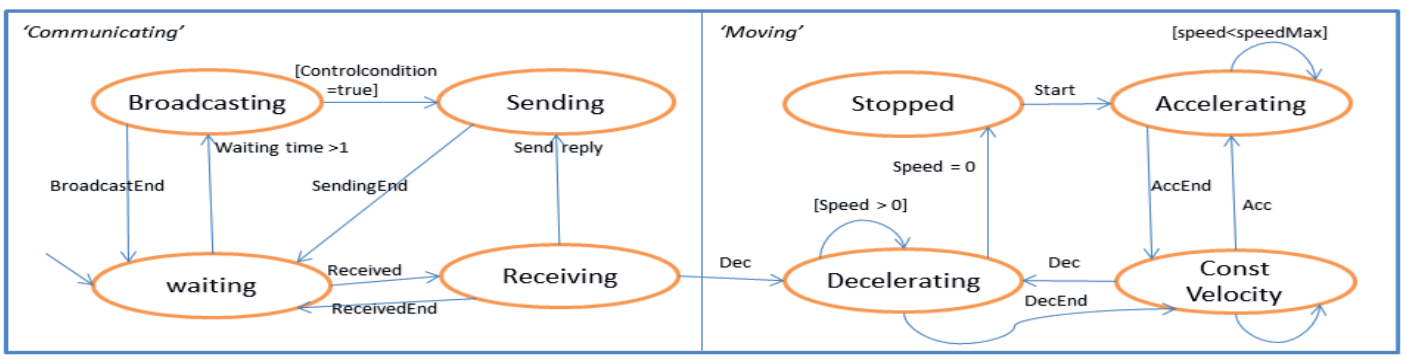

Figure 3. State transitions of IAV in communication task

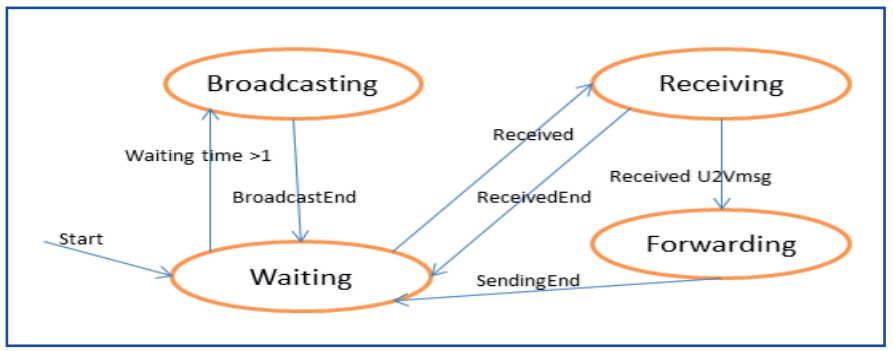

Figure 4. State transitions of UAV in communication task

\subsection{Sequence Diagram}

According to the IAV behavior described above, Figure 5 illustrates the sequence of the transmission and reception of different messages (V2V, U2V and V2U messages) established for assuring the cooperation between IAV to prevent and avoid a collision at intersections.

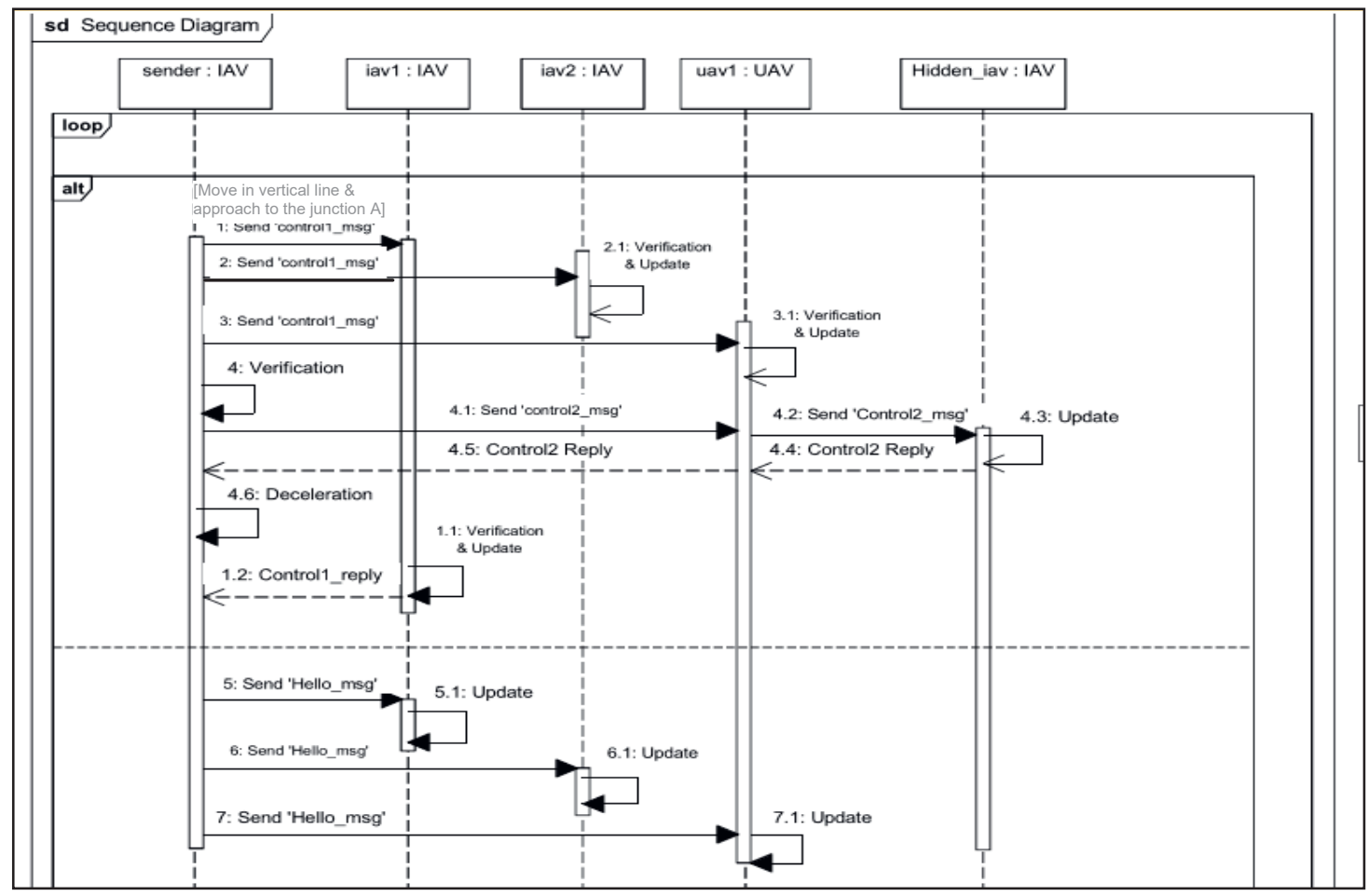

Figure 5. Sequence diagram showing the cooperation between IAV

Each IAV in the layout sends periodically 'Hello_msg' messages to inform their neighbors (IAV and UAV) about its current position. When an IAV is moving in the vertical road and approaching an intersection of type A, it checks the presence of the vehicles coming in horizontal road. This verification 
will be ensured by broadcasting the "control1_msg" messages and sending the "control2 msg" to their hidden neighbors. The UAV aids the IAV to communicate with their hidden IAV by forwarding the "control2_msg" messages and their replies.

\subsection{Distributed algorithm}

As introduced above, the proposed algorithm aims to prevent and avoid collisions at intersections. IAV communication model is focused on "Hello" message and "control" message. However, with the presence of containers stacks, V2V communication is not sufficient. The proposed algorithm is based on direct and indirect communication by implementing V2V, V2U and U2V messages. Therefore, the UAV broadcasts its neighbor's list to inform all surrounding IAV about nearly IAV to the intersection. Figure6 and Figure 7 present an abstract model of communication and cooperation at the level of IAV. Figure 8 shows how the UAV handle the received messages.

\section{At the level of IAV}

At IAV, the cooperation model is divided into two phases: discovery phase and control phase. In the discovery phase, each moving IAV periodically sends and receives beacon messages (message of type "Hello_msg"). Continually, the IAV updates the position of its neighbors in direct communication (1hop) or indirect communication (2-hop). The 2-hop neighbor (hidden IAV) is detected from the received UAV neighbors list. In otherwise, each IAV moving in a vertical line starts the control phase when it is in (d) meters or less from an intersection of type A (this is [control condition 1]). This IAV checks the presence of the approaching IAV to the same intersection in the horizontal line by sending control messages ("Control1_msg" and "Control2_msg") as presented in Figure 6. When an IAV receives a "control1 message", the verification of collision possibility at the intersection (this is [control condition $2])$ is executed. If it prevents a collision, it sends a reply message.

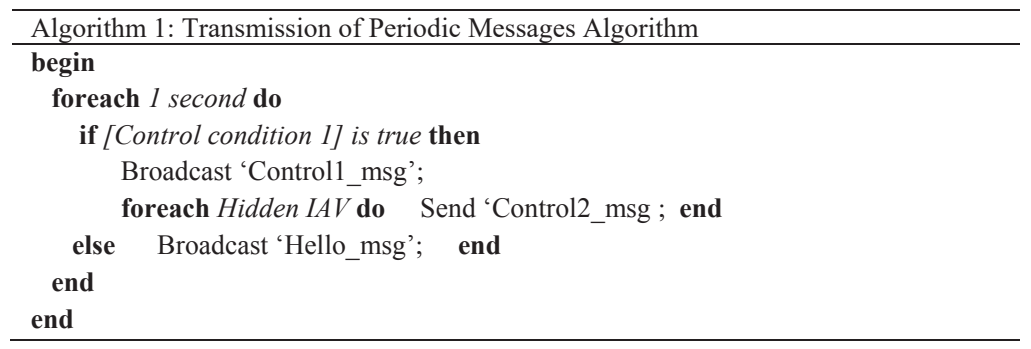

Figure 6. Transmission of periodic messages

After the reception of the control message reply, the IAV decelerates. The IAV accelerates again when the intersection is free, and there are no other approaching IAV in the high priority line (horizontal line). The control phase is finished when the IAV quits the intersection. Figure 7 shows the main steps algorithm after the reception of a $\mathrm{V} 2 \mathrm{~V}$ or $\mathrm{U} 2 \mathrm{~V}$ message.

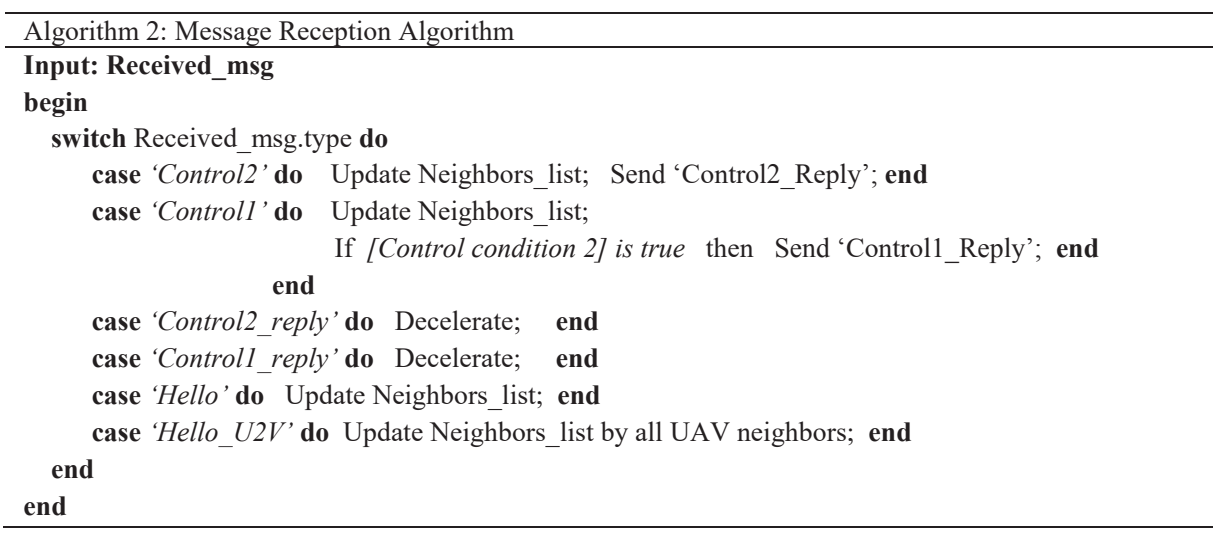

Figure 7. IAV behavior after reception of a message 


\section{At the level of $U A V$}

The UAV cooperation is divided into three tasks: 1) Broadcast periodic messages, 2) Update its neighbor's list, 3) Forward control messages and their replies. Periodically, UAV broadcasts "Hello" message (U2V message), which contains its neighbor's list. When the UAV receives "Hello" message from an IAV, it updates its neighbor's list. UAV forwards a "Control 2" or "Reply_control 2" message received from an IAV to its hidden neighbor as presented in Algorithm 3 (Figure 8).

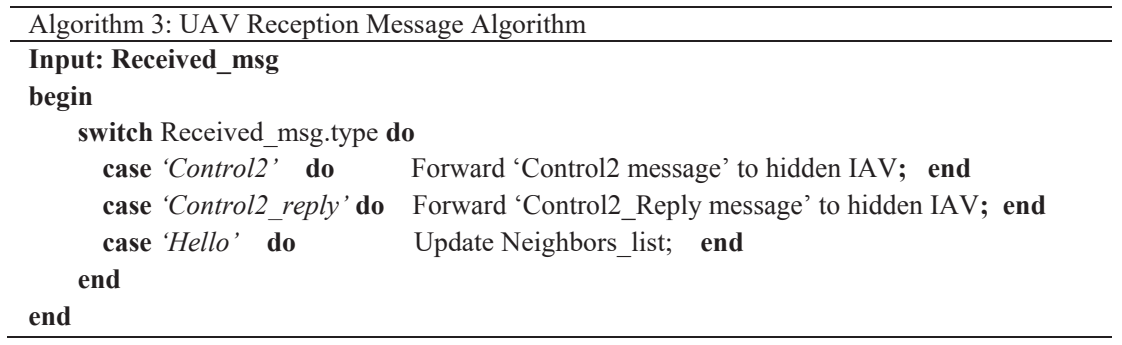

Figure 8. UAV Reception of messages

\section{Performance evaluation}

Having detailed the functionalities of our distributed algorithm, we now focus on its performance evaluation using different simulation scenarios. To study the performance, we considered the layouts defined in section 5.1. Layout 1 is formed as a single loop with one unidirectional route. Layout 2 is a set of unidirectional paths with two horizontal lanes and six vertical lines. The cross of a vertical line with a horizontal line forms an intersection. To study container processing, we have defined simulation scenarios in section 6.2.

\subsection{Simulation environment}

Different tools have been used to carry out our simulations. We investigated our proposal with veins environment simulation based on network traffic simulation (OMNET++). 'Veins' simulator is a vehicular communication tool under OMNET++ simulator which interacts with SUMO simulator.

\subsection{Simulation scenarios}

Using both layouts shown in Figure 9, we defined three scenarios: a) Scenario 1: navigation of 10 IAV in layout 1; b) Scenario 2: 10 IAV move in layout 2 by considering a signal obstruction at an intersection using a minimum required UAV as relay. In Figure 9.b, the blocs found between vertical lines represent stored containers. UAV is used here as relay node to establish the indirect communication link, which assures cooperation between IAV in the case of NLOS communication; c) Scenario 3: identical to scenario 2 but without stacks of containers in the yard. For scenario 2 and scenario 3, we predefined the navigation path of each IAV (noted $\mathrm{IAV}_{1}, \mathrm{IAV}_{2}, \ldots, \mathrm{IAV}_{10}$ ) in layout 2, as shown in Table 1. Depending on the direction of the proposed roads (see Figure 9.b), the collision can occur only at two intersections $\left(\mathrm{P}_{1}\right.$ and $\left.\mathrm{P}_{3}\right)$. For this, only two UAV are aided to the IAV network to assure safety in intersections $\mathrm{P}_{1}$ and $\mathrm{P}_{3}$.

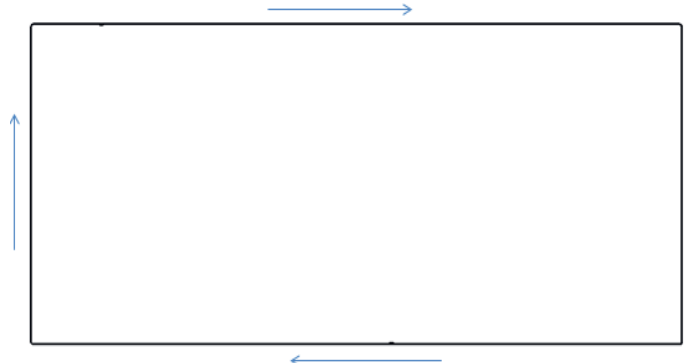

a) Layout 1

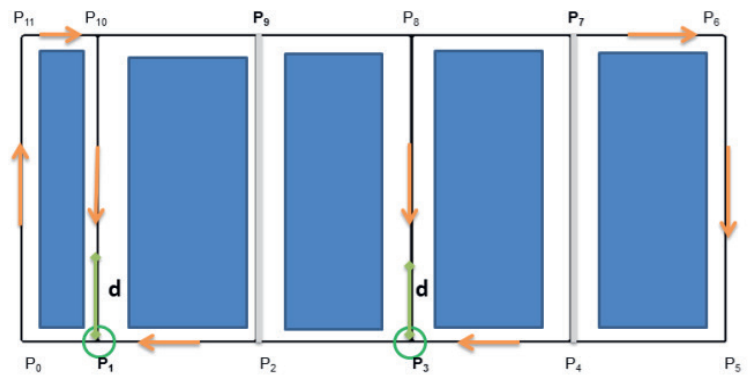

b) Layout 2

Figure 9. Schematic overview of Layouts 
Table 1. Assignment of 10 IAV in layouts 2

\begin{tabular}{|l|l|l|}
\hline $\mathbf{N}^{\circ}$ & Route & Affectation \\
\hline Route 1 & $\mathrm{P}_{0} \mathrm{P}_{11} \mathrm{P}_{10} \mathrm{P}_{1} \mathrm{P}_{0}$ & IAV $_{3}$, IAV $_{8}$, IAV $_{9}$ \\
\hline Route 2 & $\mathrm{P}_{0} \mathrm{P}_{11} \mathrm{P}_{10} \mathrm{P}_{9} \mathrm{P}_{8} \mathrm{P}_{3} \mathrm{P}_{2} \mathrm{P}_{1} \mathrm{P}_{0}$ & IAV $_{4}$, IAV $_{5}$, IAV $_{6}$, IAV $_{7}$, IAV $_{10}$ \\
\hline Route 3 & $\mathrm{P}_{0} \mathrm{P}_{11} \mathrm{P}_{10} \mathrm{P}_{9} \mathrm{P}_{8} \mathrm{P}_{7} \mathrm{P}_{6} \mathrm{P}_{5} \mathrm{P}_{4} \mathrm{P}_{3} \mathrm{P}_{2} \mathrm{P}_{1} \mathrm{P}_{0}$ & IAV $_{1}$, IAV $_{2}$ \\
\hline
\end{tabular}

\subsection{Simulation parameters}

This study aims to avoid collision between IAV at the junction when moving in different routes and approaching the same junction. For this, we make some assumptions. All moving vehicles emit "Hello messages" every 1 second. We also assume that each IAV can determine its current position in the layout. The initial position of each IAV is $(0,0)$. The simulation time is the end of the working time of all IAV. Container handling time between IAV and crane (QC and ASC) is ignored in all simulations. We place $U A V_{1}$ and $U A V_{2}$ over the intersection $P_{1}$ and $P_{3}$ for the second layout, respectively. The IAV moving in the vertical line starts the control phase when it is less than $\boldsymbol{d}=\mathbf{5 0}$ meters from the intersection. This phase is finished when the IAV quits the intersection. Table 2 presents simulation parameters. We randomly set the start time of each IAV in all simulations, as shown in Table 3.

Table 2. Simulation parameters

\begin{tabular}{|l|l|l|}
\hline Parameter & Default value & Proposed value \\
\hline IAV number & 10 & - \\
UAV number & 2 & $0,1,2$ \\
UAV higher & $30 \mathrm{~m}$ & $0 . .30$ \\
Container number & 10 & $10 \ldots 300$ \\
Layout dimension & $900 \mathrm{~m}^{*} 400 \mathrm{~m}$ & - \\
Inter-IAV distance (minGap) & $2 \mathrm{~m}$ & - \\
Interval Broadcast & $1 \mathrm{~s}$ & $1 \mathrm{~s} \ldots . .10 \mathrm{~s}$ \\
Max Speed of IAV & $7 \mathrm{~m} / \mathrm{s}$ & - \\
Max acceleration & $0.5 \mathrm{~m} / \mathrm{s}^{2}$ & - \\
Max deceleration & $2.5 \mathrm{~m} / \mathrm{s}^{2}$ & - \\
Transmission power & $10 \mathrm{~mW}$ & $1 \mathrm{~mW} \ldots 20 \mathrm{~mW}$ \\
Technology & $5.89 \mathrm{Ghz}$ & - \\
Distance of control (d) & $50 \mathrm{~m}$ & - \\
\hline
\end{tabular}

Table 3. IAV start time

\begin{tabular}{|l|l|l|l|l|l|l|l|l|l|l|}
\hline IAV & IAV1 & IAV2 & IAV3 & IAV4 & IAV5 & IAV6 & IAV7 & IAV8 & IAV9 & IAV10 \\
\hline Start time (s) & 0 & 6 & 40 & 115 & 121 & 130 & 136 & 143 & 149 & 170 \\
\hline
\end{tabular}

\subsection{Performance metrics}

"Veins" simulator has been used in the following experiments to measure the efficiency of the proposed cooperation model in terms of the number of detected and avoided collisions in layout 2 and the number of received and sent messages. We report only "control" messages transmitted among the IAV and UAV. We record the number of the lost packets by varying the height of UAV. We, therefore, compare the handling containers in layout 1 and layout 2 (scenario 1 and scenario 2) by recording the following indicators: i) Total discharging time (the completion time for handling all containers); ii) IAV average operation time (the average time that an IAV will spend to assure request work); iii) End working time; iv) IAV activity in layout 1 and layout 2 (where each IAV handles 10 containers); v) Short working time of IAV.

\subsection{Result analysis}

- Collision detection and avoidance

Figure 10.a and Figure 10.b show that the number of detected and avoided collisions is related to the number of handled containers in scenario 2. This result indicates that the proposed cooperative mechanism is efficient in preventing and avoiding collisions at an intersection. Handling of an important number of containers results in more movement of IAV in the layout where the occurrence of collision situations increases. Collision number also depends on IAV affectation. In the case of signal obstructions, direct communication is not enough. The communication in the IAV network is reliable only when the inter-IAV communication is combined with indirect communication based on UAV. Indirect communication is useful for continuous cooperation between IAV to manage the intersection access. 


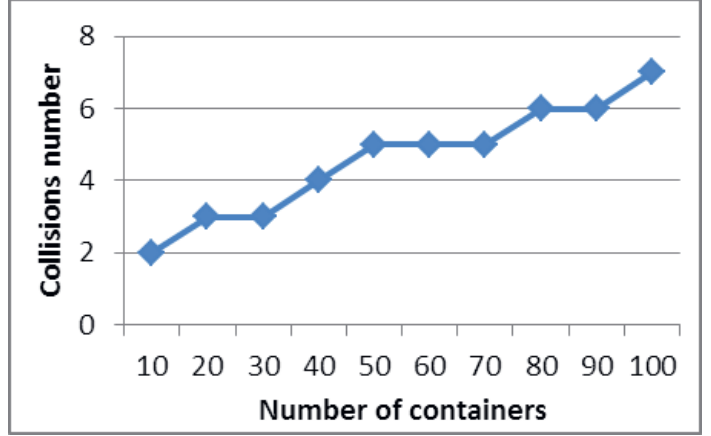

(a)

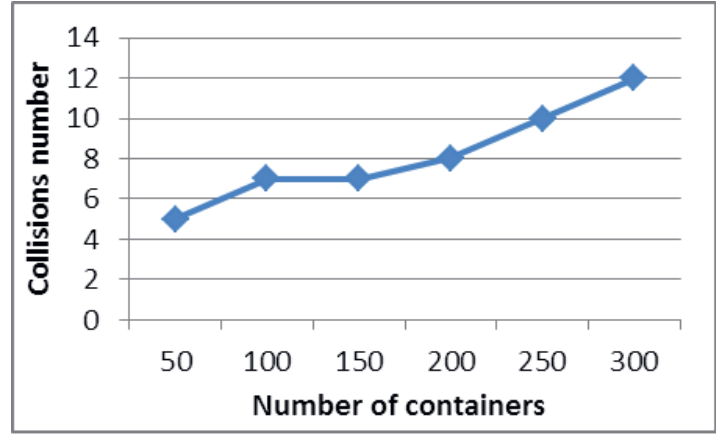

(b)

Figure 10. Collisions number vs. containers number

Simulation of scenario 3 gives the same result in terms of avoidance collisions. In this case, the cooperation between IAV is assured only by V2V communication. With the presence of LOS communication between IAV at intersections, the relay node (UAV) is not useful.

- Received and sent V2U message

Figure 11 presents the number of V2U control messages received by UAV and the number of V2U control messages sent by IAV to UAV in the simulations of scenario 2 under different number of containers from 10 to 100 with an increment of 10 containers (Figure 11.a and Figure 11.c) and from 50 to 300 containers with an increment of 50 containers (Figure 11.b and Figure 11.d). As presented in sequence diagram (Figure 5), these messages are of "Control 2" type. We result that the total of V2U control messages sent by IAV is equal to the total of V2U control messages received by UAV. Each sent V2U control message is received by a UAV which forwards it to the hidden IAV as indicated in the control message. This fact demonstrates that during the simulation, there are many NLOS situations where the IAV require the assistance of UAV to cooperate with each other to avoid collisions. When an IAV receives U2V control message, it sends via a UAV a reply of this control message. Each UAV in its location forwards the received V2U message (control or reply) to the designed destination. Figure 12 represents the forwarded V2U message by UAV in different simulations.

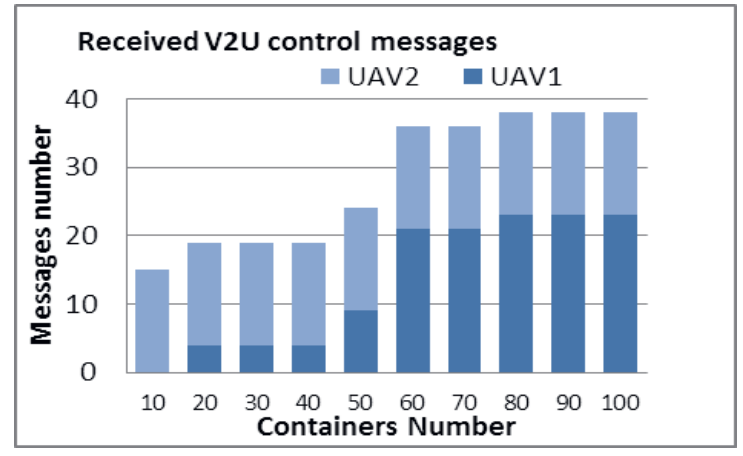

(a)

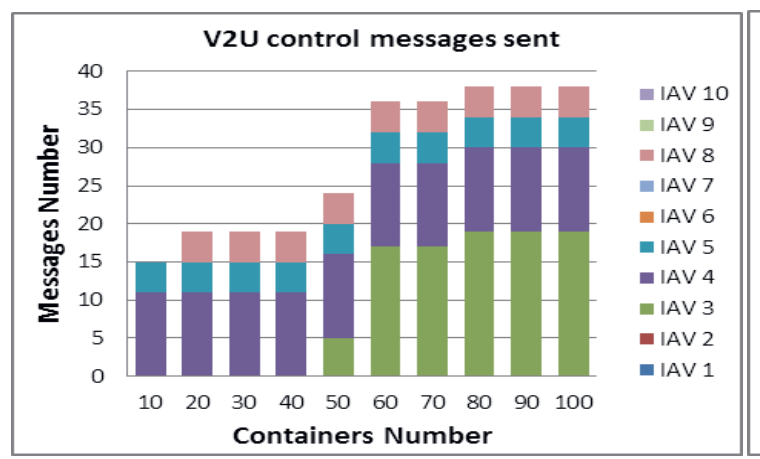

(c)

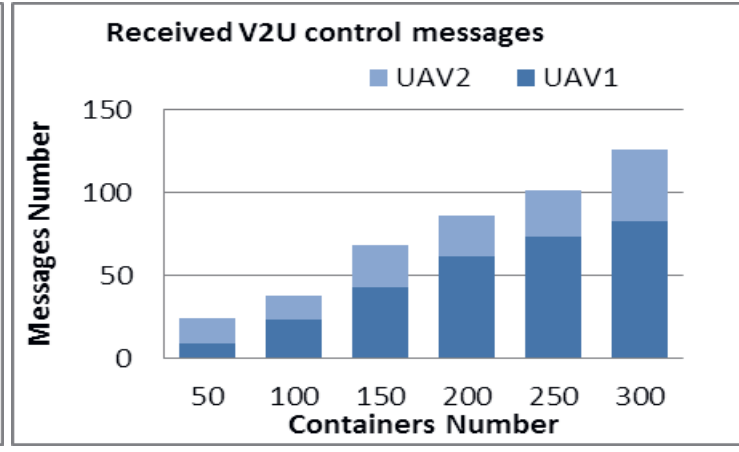

(b)

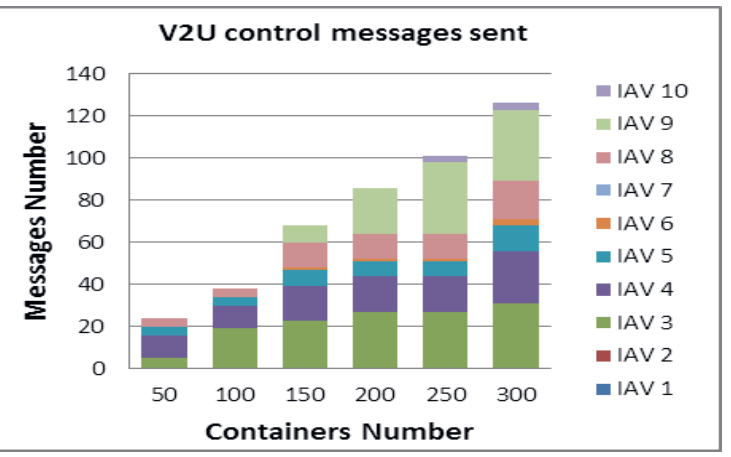

(d)

Figure 11. V2U control messages vs containers number 


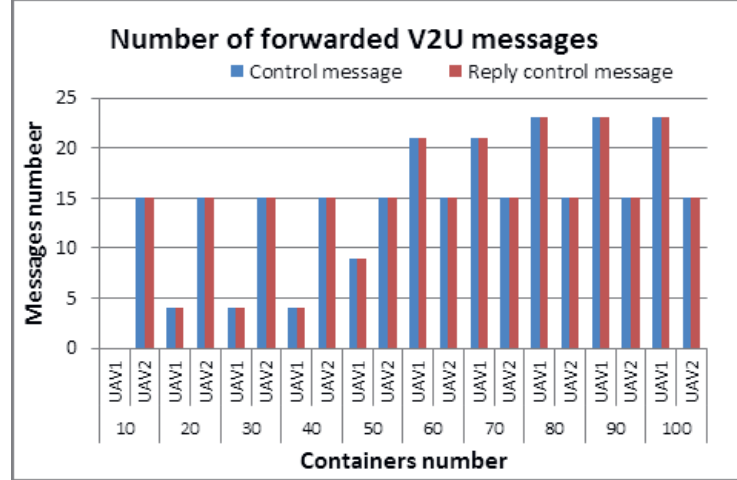

(a)

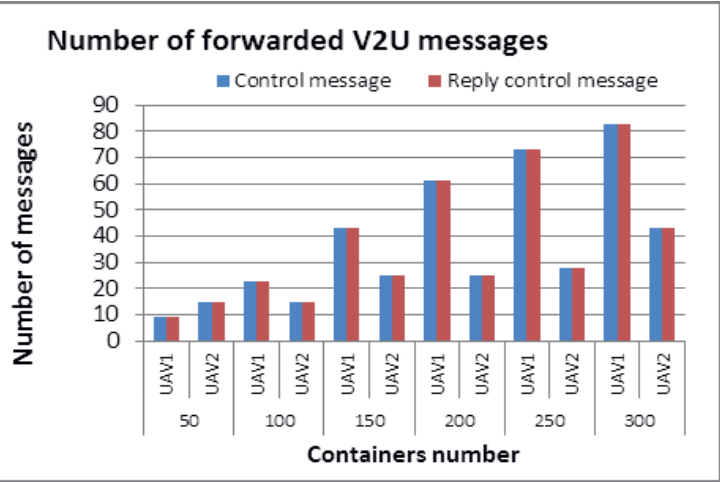

(b)

Figure 12. Forwarded V2U messages vs containers number

- Comparison between scenario 1 and scenario 2

Figure 13 shows the simulations result regarding the operating time (start time and stop time) of ten IAV for handling 50,100,150, 200, 250, and 300 containers. The operating time is related to the number of handled containers and the length of route assigned (Route 1: IAV $V_{3}, I A V_{8}$, and IAV $V_{9}$ : Route 2: $I A V_{4}, I A V_{5}, I A V_{6}, I A V_{7}$ and $I A V_{10}$ and Route 3: IAV $V_{1}$ and $I A V_{2}$ ). In layout 2, the average operating time reaches the minimum operating time, contrary to layout 1 where the working time of IAV is almost equal to simulation time.

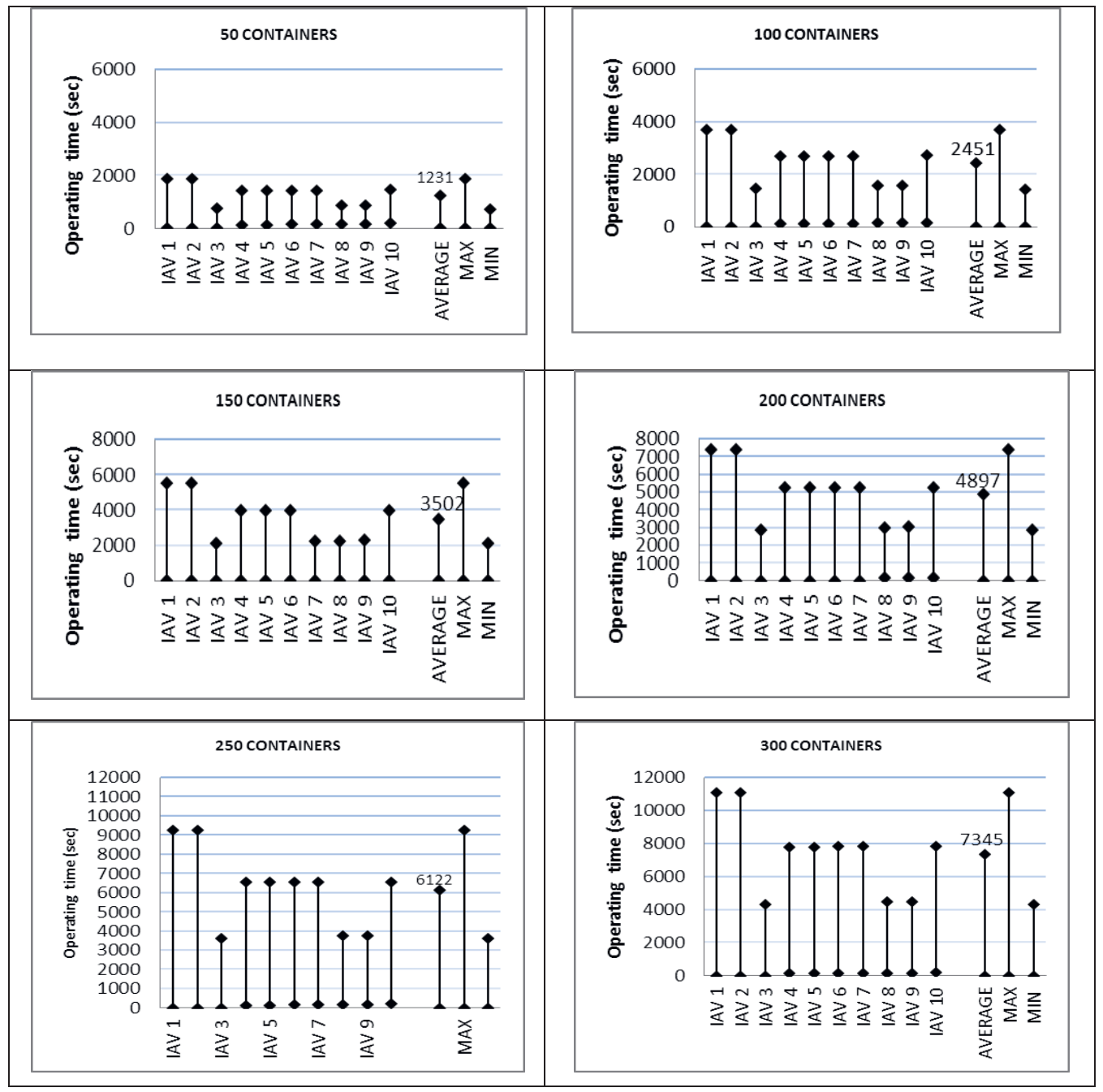

Figure 13. Operating time vs containers number 
Figure 14 plots IAV operating rate for the handling of 50, 100, 150, 200, 250, and 300 containers in scenario 2 . We observe that the operating rate is $38 \%, 69 \%$, and $99 \%$ for an IAV moved in routes 1,2 and 3 , respectively. In scenario 1 , the operating rate reaches $99 \%$ for all IAV.

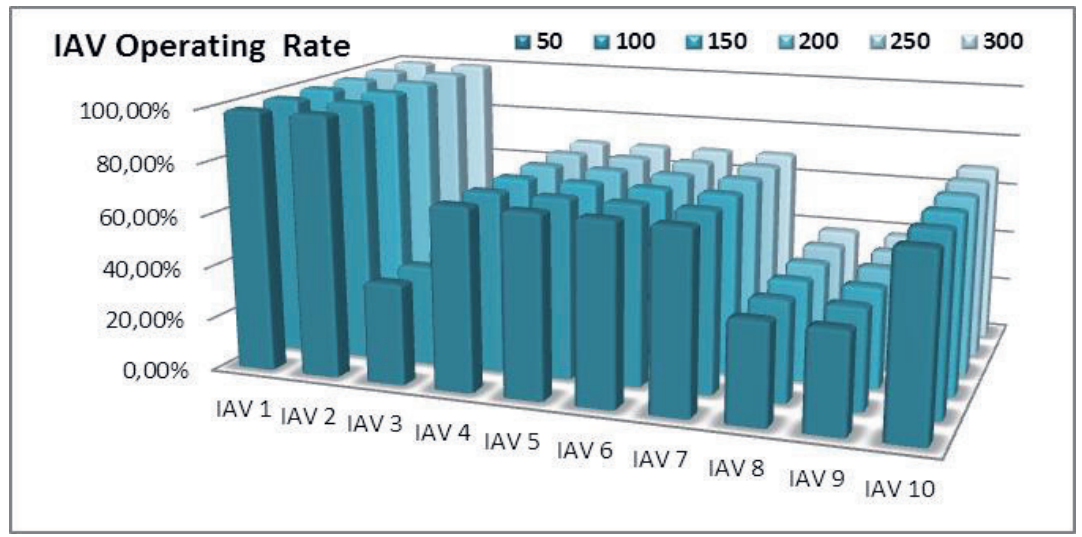

Figure 14. IAV Operating rate vs containers number

Figure 15 presents a comparison between scenario 1 and scenario 2 in terms of the total time of each IAV in handling 100 containers. For example, we observe that IAV3, IAV8, and IAV9 are quickly released in scenario 2 (and scenario 3). These IAV are affected by the short route (Route 1). Figure 16 shows a comparison between scenario 1 and scenario 2 regarding the stop time of the first IAV that terminates its operation in the handling task. Contrary to scenario 1, some IAV finished their tasks quickly in scenario 2 and scenario 3.

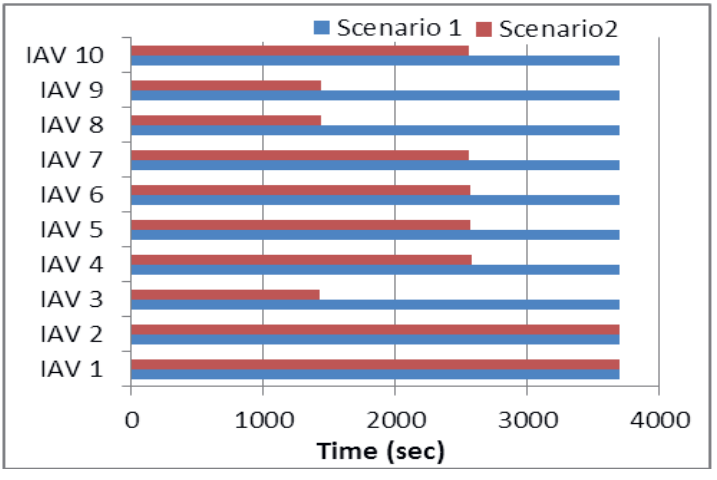

Figure 15. Total time of ten IAV (handling 100 containers)

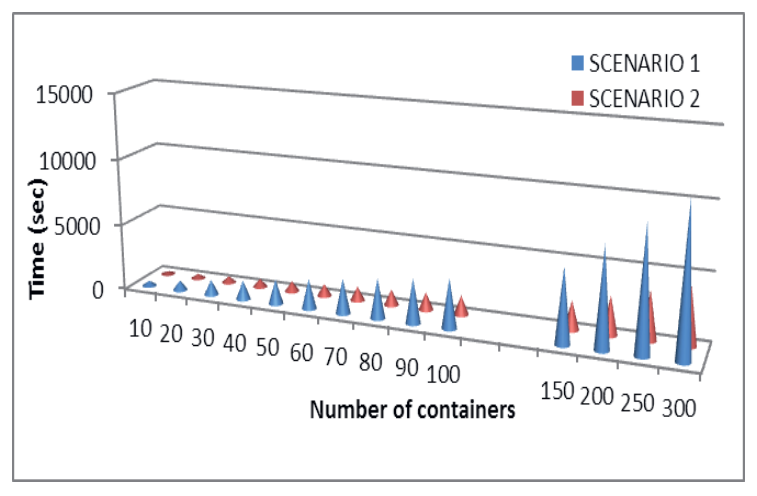

Figure 16. IAV first end-time

Figure 17 presents IAV average operating time in both scenarios. The average operating time is $33 \%$ more in scenario 1 compared to scenario 2. Furthermore, Figure 18 illustrates the average operating time per container.

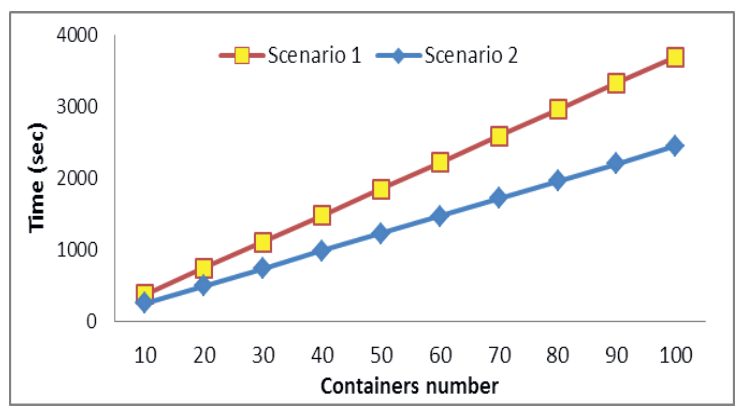

Figure 17. IAV Average operating time vs containers number

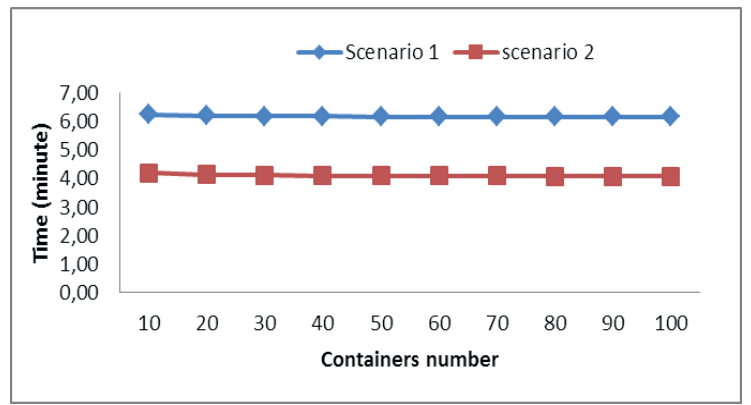

Figure 18. IAV average operating time per container vs containers number 
- The influence of UAV height

We study the effect of the UAV altitude. Figure 19 presents the number of lost packets in the network according to the number of containers in UAV altitude of 20 meters.

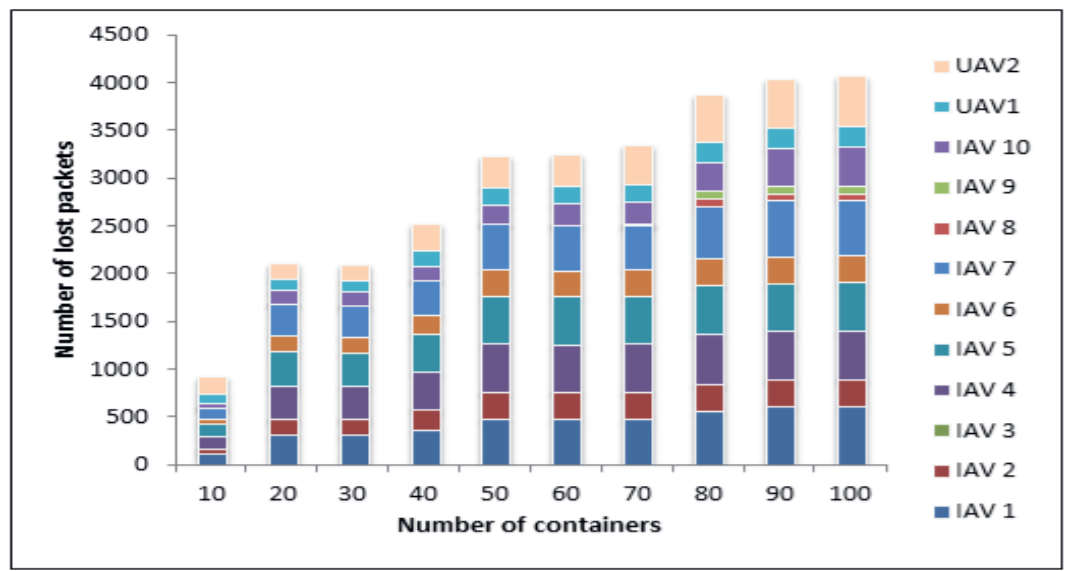

Figure 19. Lost packets number vs containers number

Figure 20 illustrates the total number of lost packets according to the number of containers for different UAV altitudes $(0,10,20$, and $30 \mathrm{~m})$. In this simulation, almost all collisions are detected and avoided. But we report that the number of the lost packets increases according to the number of the containers when the UAV height is less than 30 meters. In the simulation carried out, the lost packets are mainly of the "Hello_msg" type. This case indicates that the cooperation between IAV has to find a compromise UAV altitude.

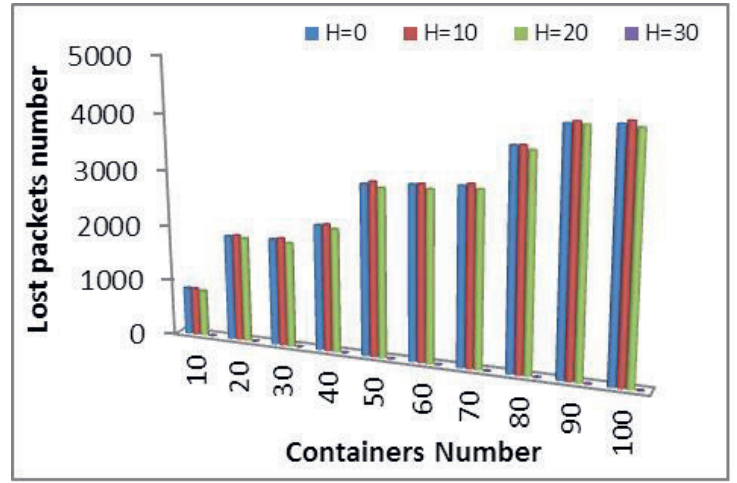

(a)

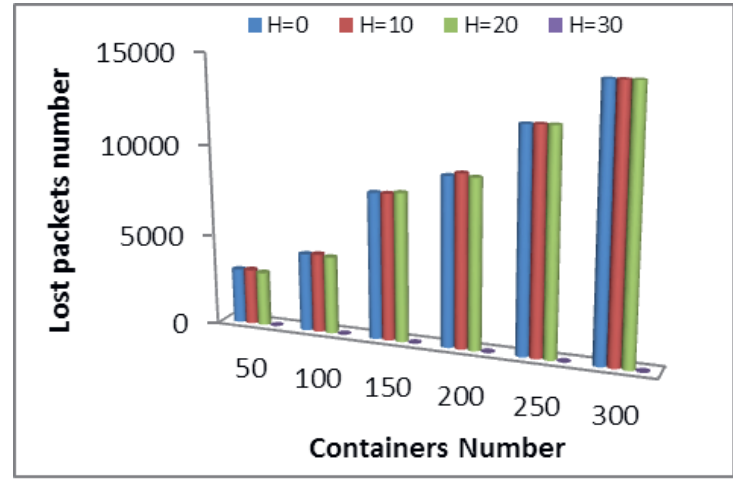

(b)

Figure 20. Lost packets number vs UAV height

\section{Conclusion}

This paper investigated IAV cooperation for AIM based on direct/indirect communication using relay nodes. In our study, the relay node is UAV. The proposed relay strategy can efficiently improve communication reliability and thus ensure cooperation inter-IAV. The principal novelty of this work is the use of UAV as a communication relay in a port environment by considering the signal obstruction. We, therefore, conclude that IAV cooperation stays efficient even in the case of signal obstruction where the indirect communication between IAV is provided by UAV deployed on intersections. UAV nodes are primordial elements for reliable communication in a harsh and dynamic environment. They prove to be very helpful in supporting message exchange between ground nodes at any given time. We have provided communication models validated by simulations of an automatic control system for preventing and avoiding collision between IAV when crossing at an intersection. Prevent and avoid collision contribute to the continuance of service in ACT. The simulations have confirmed the effectiveness of our proposal. As future works, we plan to study the use of UAV as automated manager stations in the case of shadowing signals like in ACT. 


\section{References}

1. Ahmane, M., Abbas-Turki, A., Perronnet, F., Wu, J., EL Moudni, A., Buisson, J. and Zeo, R. (2013) Modeling and controlling an isolated urban intersection based on cooperative vehicles. Transportation Research Part C, 28, 44-62.

2. Ambroziak, S. J., and Katulski, R. J. (2014) Characteristics of propagation conditions in the container terminal environment. In: International Symposium on Electromagnetic Compatibility, May 2014, Tokyo: IEEE, 701-704.

3. Azimi, R., Bhatia, G., Rajkumar, R., and Mudalige, P. (2012) Intersection management using vehicular networks, SAE Tech. Papers 2012-01-0292, 6(2), 681-690.

4. Bahnes, N., Kechar, B., and Haffaf, H. (2016) Cooperation between Intelligent Autonomous Vehicles to Enhance Container Terminal Operations. Elsevier Journal of Innovation in Digital Ecosystems, 3(1), 22-29.

5. Belmekki, B. E. Y., Hamza, A., and Escrig, B. (2019) Cooperative vehicular communications at intersections over nakagami-m fading channels. Vehicular Communications, 19, 1-11. doi:10.1016/j.vehcom.2019.100165.

6. Campos, G. R., Falcone, P., Hult, R., Wymeersch, H., and Sjöberg, H. (2017) Traffic coordination at road intersections: Autonomous decision-making algorithms using model-based heuristics. IEEE Intelligent Transportation System Magazine, 9(1), 8-21. doi: 10.1109/MITS.2016.2630585.

7. Chen, W., Guha, R. K., Kwon, T. J., Lee, J., and Hsu, Y. Y. (2011) A survey and challenges in routing and data dissemination in vehicular ad hoc networks. WIRELESS COMMUNICATIONS AND MOBILE COMPUTING, 11, 787-795.

8. Chu, F., Gailus, S., Liu, L., and Ni, L. (2018) The future of automated ports. (Available in https://www.mckinsey.com).

9. Dong, M., Ota, K., Lin, M., Tang, Z., Du, S., and Zhu, H. (2014) UAV-assisted data gathering in wireless sensor networks. Journal of Supercomputing, 70, 1142-1155.

10. Dresner, K., and Stone, P. (2004) Multi-agent traffic management: A reservation-based intersection control mechanism. In: Proceedings of the $3^{\text {rd }}$ International Joint Conference on Autonomous Agents and Multiagent Systems, July 2004, USA: IEEE Computer Society, 530-537.

11. Eckhoff, D., Sommer, C., German, R., and Dresseler, F. (2011) Cooperative Awareness at Low Vehicle Densities: How Parked Cars Can Help See Through Buildings. In: 2011 IEEE Global Telecommunications Conference, December 2011, USA: IEEE, 1-6.

12. Elhenawy, M., Elbery, A. A., Hassan, A. A., and Rakha, H. A. (2015) An Intersection Game Theory Based Traffic Control Algorithm in a Connected Vehicle Environment. In: 2015 IEEE 18th International Conference on Intelligent Transportation Systems, September 2015, Spain: IEEE, 343-347.

13. Erdelj, M., Natalizio, E., Chowdhury, K. R., and Akyildiz, I. F. (2017) Help from the Sky: Leveraging UAVs for Disaster Management. PERVASIVE computing, 16(1), 24-32.

14. Floreano, D., and Wood, R. J. (2015) Science, technology and the future of small autonomous drones. Nature, 521(7553), 460-466. https://doi.org/10.1038/nature14542.

15. Gelareh, S., Merzouki, R., McGinley, K., and Murray, R. (2013) Scheduling of intelligent and autonomous vehicles under pairing/unpairing collaboration strategy in container terminals. Transportation Research part C, 33, 1-21.

16. Guenther, H., Grunow, M., Lehmann, M., Neuhaus, U., and Yilmaz, I. O. (2006) Simulation of transportation activities in automated seaport container terminals. In: Proceedings of the $2^{\text {nd }}$ International Intelligent Logisctics System Conference, February 2006, Australia, 1-15.

17. Hafner, M. R., Cunningham, D., Caminiti, L., and Del Vecchio, D. (2013) Cooperative collision avoidance at intersections: Algorithms and experiments. IEEE Transactions on Intelligent Transportation System, 14(3), 1162-1175.

18. Hassanalian, M., and Abdelkefi, A. (2017) Classifications, applications, and design challenges of drones: A review, Progress in Aerospace Sciences, 91, 99-131. https://dx.doi.org/10.1016/j.paerosci 2017.04.003.

19. Kechar, B., and Haffaf, H. (2012) Communication Architecture Based on Intelligent Autonomous Vehicles for Container Terminals. In: $12^{\text {th }}$ International Conference on ITS Telecommunications, November 2012, Taiwan: IEEE, 769-774.

20. Lin, Y., Wang, P., and Ma, M. (2017) Intelligent Transportation System (ITS): Concept, Challenge and Opportunity. In: 2017 IEEE $3^{\text {rd }}$ international conference on big data security on cloud (big data security), IEEE International Conference on High Performance and Smart Computing (HPSC), and 
IEEE International Conference on Intelligent Data and Security (IDS), May 2017, Beijing: IEEE, 167-172. doi: 10.1109/BigDataSecurity.2017.50.

21. Lombard, A., Perronnet, F., Turki. A.A., and Moudni, A. (2016) Decentralized management of intersections of AGVs. IFAC-PapersOnLine Elsevier, 49(12), 497-502. https://doi.org/10.1016/j.ifa col.2016.07.669.

22. Martín-Soberón, A. M., Monfort, A., Sapiña, R., Monterde, N., and Calduch, D. (2014) Automation in port container terminals. Procedia - Social and Behavioral Sciences, 160, 195-204.

23. Melin, J., Lauri, M., Kolu, A., Koljonen, J., and Ritala, R. (2015) Cooperative Sensing and Path Planning in a Mult-vehicle Environment. IFAC-PapersOnLine (2015) Elsevier, 48(9), 198-203. 10.1016/j.ifacol.2015.08.083.

24. Noor, N. M., Abdullah, A., and Hashim, M. (2018) Remote sensing UAV/drones and its applications for urban areas: a review. IOP Conference Series. Earth Environmental Science, 169(1), 1-8. https://doi.org/10.1088/1755-1315/169/1/012003.

25. Oubbati, O. S., Lakas, A., Lagraa, N., and Yagoubi, M. B. (2015). CRUV: Connectivity-based Traffic Density Aware Routing using UAVs for VANets. In: International Conference on Connected Vehicles and Expo (ICCVE), October 2015, China: IEEE, 68-73.

26. Oubbati, O.S., Lakas, A., Lagraa, N., and Yagoubi, M. B. (2016) UVAR: An Intersection UAVAssisted VANET Routing Protocol. In: Proceeding IEEE Wireless Communications and Networking Conference (WCNC 2016), September 2016, USA: IEEE, 1-6.

27. Ranjan, A., Panigrhi, B., Sahu, H. B., and Misra, P. (2018) SkyHelp: UAV Assisted Emergency Communication in Deep Open Pit Mines. In: Proceedings of the $1^{\text {st }}$ International Workshop on Internet of People, Assistive Robots and Things, June 2018, Germany: ACM, 31-36.

28. Ruban, I., and Zhang, R. (2007) Placement of UAVs as Communication Relays Aiding Mobile Ad Hoc Wireless Networks. In: IEEE Military Communications Conference (MILCOM2007), October 2007, USA: IEEE, 1-7.

29. Sahingoz, O. K. (2013) Mobile Networking with UAVs: Opportunities and Challenges. In: 2013 International Conference on Unmanned Aircraft Systems (ICUAS), May 2013, USA: IEEE, 933-941.

30. Shi, W., Zhou, H., Li, J., Xu, W., Zhang, N., and Shen, X. (2018) Drone Assisted Vehicular Networks: Architecture, Challenges and Opportunities. IEEE Network, 32(3), 130-137.

31. Sommer, C., Eckhoff, D., and Dressler, F. (2014) IVC in Cities: Signal Attenuation by Buildings and How Parked Cars Can Improve the Situation. IEEE TRANSACTIONS ON MOBILE COMPUTING, 13(8), 1733-1745.

32. Tonguz, O. K., and Viriyasitavat, W. (2016) A Self-Organizing Network Approach to Priority Management at Intersections. IEEE Communication Magazine, 54(6), 119-127.

33. URL1 (2021) Camco technologies: https://www.camco.be/ (Last accessed: 30/07/2021).

34. URL2 (2020) Port-news: https://www.joc.com (Last accessed: 12/08/2020).

35. URL3 (2020) Rotor-copters example: https://www.rotorcopters.com/walkera-qr-x800-quadcopter/ (Last accessed: 19/07/2020).

36. Vásárhelyi, G., Virágh, C., Somorjai, G., and Nepusz, T. (2018) Optimized flocking of autonomous drones in confined environments. Science Robotics, 3(20), 1-14.

37. Wang, X., Fu, L., Zhang, Y., Gan, X., and Wang, X. (2016) VDNet: An infrastructure-less UAVassisted sparse VANET system with vehicle location prediction. Wireless Communication and Mobile Computing, 16(17), 2991-3003.

38. Yang, X., Liu, L., Vaidya, N. H., and Zhao, F. (2004) A vehicle-to-vehicle communication protocol for cooperative collision warning. The First Annual International Conference on Mobile and Ubiquitous Systems: Networking and Services (MOBIQUITOUS 2004), August 2004, USA (Boston, MA): IEEE, 114-123.

39. Yu, T., Zhao, J., and Gong, Y. (2019) UAV aided Localization algorithm relay for train mounted mobile terminals. Physical communication, 34, 227-234. 